\title{
A constructive peridynamic kernel for elasticity
}

\author{
Ziguang Chen, Drew Bakenhus and Florin Bobaru* \\ Mechanical and Materials Engineering, University of Nebraska-Lincoln, Lincoln, NE, 68588- \\ 0526 USA
}

Different kernel formulations have been proposed and used for bond-based peridynamic models of elastic materials. While all such kernels produce results that, in the limit of the nonlocal region (horizon) going to zero (while grid spacing relative to the horizon size is constant), converge, not all converge to the classical solution. Here, we introduce a constructive path to arriving at a peridynamic kernel for 1D, 2D and 3D elasticity. We focus on the particular one-point Gauss quadrature method of spatial discretization and study the convergence properties of different kernels with respect to the classical, local, model for dynamic elasticity in $1 \mathrm{D}$, where exact representation of geometry is possible. We analyze the influence of two ways of imposing boundary conditions and of the "skin effect" on the solution. We show that, similar to the diffusion case, the peridynamic kernel derived based on physical principles for dynamic elasticity is the only one whose convergence to the classical solution does not depend on the fineness of the discretization grid, relative to the nonlocal region size. The results presented demonstrate that special care must be taken when claiming validation of peridynamic results by specific comparisons with classical, local model solutions.

Keywords: peridynamics; dynamic elasticity; nonlocal model; convergence; nonlocal dispersion; wave propagation.

*Corresponding author. Tel.: +1 402472 8348; Email: fbobaru2@unl.edu 


\section{Introduction}

The classical theory of solid mechanics contains spatial derivatives, which may not exist when discontinuities in displacement, such as cracks appeared in the elastic body. The traditional way to deal with this issue is through special techniques that have been developed in the field of computational fracture mechanics [1]. These special techniques are not always satisfactory, either physically or mathematically, in part because of the need for supplemental relations that control crack growth. The peridynamic (PD) theory, introduced by Stewart Silling as a reformulation of the classical continuum mechanics [2], is an integral-type nonlocal model that allows fracture and damage to be treated as natural parts of the solution process. The formulation is based on nonlocal interactions between material points in a continuum. The PD interaction between a material point and its neighbors extends beyond the nearest neighbors, over a region called "the horizon" [2]. A fundamental generalization of the original peridynamic theory beyond pair-wise interaction between material points was published in [3]. A review of peridynamics is given in [4]. This method is particularly well suited for dealing with cracks and damage in solid mechanics especially in situations where the crack path, for example, is not known in advance [5-7]. Apart from dynamic fracture, the PD theory has been successfully applied in modeling heat transfer (in bodies with evolving discontinuities) [8], dielectric breakdown, and corrosion damage $[9,10]$ among other topics. The main reasons for which PD models are able to perform well in such problems, for example, are the removal of spatial derivatives from the formulation and the way damage is defined in the model. These two features allow for generation and propagation of strong discontinuities in the model without the mathematical and algorithmic complications present in classical models, such as the local elasticity model discretized with the finite element method. These characteristics are well suited for a meshfree-type discretization that permits autonomous growth of damage/cracks at any location and in any direction as directed by the stress and strain fields present in the body.

In this paper we discuss the selection of the kernel of the integral in the PD formulation for dynamic elasticity. This is along the lines of our recent contribution on kernel selection for diffusion-type problems [11]. We point to differences and similarities to the diffusion type problems. We provide a constructive approach for deriving a PD kernel, and then compare its behavior with other kernels used in the literature $[12,13]$.

To construct a PD elasticity model for a body $\Omega$, we consider a horizon $H_{x}$ around point $x$ (see 1D case in Figure 1), the point $x$ is characterized by its displacement, $u(x, t)$. Mechanical bonds connect $x$ with all $\hat{x}$ in $H_{x}$. In the bond-based PD, the assumption is that the mechanical force exerting in the $(x, \hat{x})$ bond is independent from the force in the rest of the bonds, while in the state-based formulation, the force through $(x, \hat{x})$ is directly influenced by the rest of the bonds within the horizon region $H_{x}$. In this paper we follow the bond-based formulation, which is a particular case of the state-based PD (see [3]). The mechanical bond between $x$ and $\hat{x}$ is characterized by an elastic modulus-like quantity $c(\hat{x}, x)$, refer to as the bond "micro-modulus". The peridynamic equations of motion for bond-based model are given as: 
$\rho \frac{\partial^{2} u(x, t)}{\partial t^{2}}=\int_{H_{x}} f(\hat{x}, x, t) \mathrm{d} V_{\hat{x}}$

where $\rho$ is the mass density and $f$ is the pairwise force function in the peridynamic bond connecting point $\hat{x}$ to $x$, and $u$ is the displacement. In this formulation, we ignore body forces. The integrand (the function under the integral sign in Eq. (1)), the force-like quantity $f$, is usually taken to be of the form (see [12-17]):

$f_{n}(\hat{x}, x, t)=\left\{\begin{array}{c}c(\hat{x}, x) \frac{u(\hat{x}, t)-u(x, t)}{|\hat{x}-x|^{n}},|\hat{x}-x| \leq \delta \\ 0, \quad|\hat{x}-x|>\delta\end{array}\right.$

where $\delta$ is the horizon size (in $1 \mathrm{D}, \delta$ is half the length of the interval $H_{x}$; in 2D, $\delta$ is the radius of the disk $H_{x}$ centered at $x$, for a circular choice of the horizon region; in $3 \mathrm{D}$, is the radius of the sphere $H_{\boldsymbol{x}}$ centered at $\boldsymbol{x}$, for a spherical choice of the horizon region.). Here $n$ is an integer, normally selected to be 0,1 , or 2 (see, e.g. [8, 12-14]). In the context of the mechanical peridynamic model, the $c(\hat{x}, x) /|\hat{x}-x|^{n}$ portion of the integrand is referred to as the peridynamic kernel.

The PD equations contain differentiation with respect to time, and integration over the spatial domain (the horizon region). They do not contain any spatial derivatives of the primary variable (displacement in the case of elasticity); thus, the PD equation is valid everywhere whether or not discontinuities in the displacement exist.

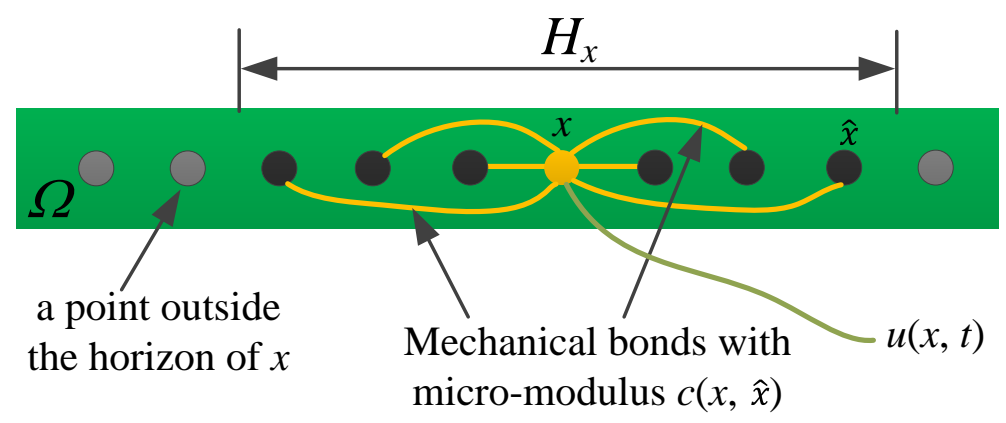

Figure 1. Peridynamic description of the elasticity model. The region denoted by $H_{x}$ is the horizon of point $x$.

Different values of $n$ in the representation of the PD kernel function for elasticity have been selected in the past. The value $n=1$ is used in [13], and this value has been used most often for the mechanical PD formulation (see e.g. [12, 18-20]). The value $n=0$ has been suggested in [21]. The latter two cases are postulated forms of the kernel. A constructive approach to derive the kernel function for diffusion problems in PD leads to $n=2$ (see $[8,14]$ ). In [11], we analyzed the behavior of peridynamic solutions for transient heat/mass diffusion relative to the form of the kernel used for a specific numerical spatial integration scheme, the one-point Gaussian quadrature. We found that only for the kernel from the constructive approach with $n=2$, the 
numerical quadrature is exact. Therefore, out of three types of kernels used in the literature, only the constructive one leads to results that converge to the classical solution in the limit of the horizon size going to zero when the relative grid-density is kept constant (this is known as $\delta$ convergence, see [14]). For all other kernels, $\delta$-convergence happens but to different values than the expected, classical ones. We also noticed that for the constructive kernel, when two " $m$ convergence" curves corresponding to two different horizon sizes for the solution at a point and at a certain time intersect, the intersection point is also the value of the exact classical solution. We attributed the crossing of $m$-convergence curves with the classical solution to the "competition" between the way boundary conditions are imposed and nonlocality (see [11]). The infinite propagation speed in the parabolic type PD diffusion equation means that the boundary conditions are felt instantly over the entire computational domain at all times. In dynamic elasticity, where the propagation speed is finite, it is foreseen that the way boundary conditions are imposed affects the displacement evolution non-uniformly in time, and the $m$-convergence curves for the PD elasticity model might not cross at a single point.

In this paper, we provide a constructive approach finding the PD kernel. The derivation is for $1 \mathrm{D}, 2 \mathrm{D}$ or $3 \mathrm{D}$. We then study the properties of different kernels with respect to convergence to the classical, local, model for dynamic elasticity equation in 1D. We select the quadrature method to be the one-point Gauss integration, and try to determine which kernel is appropriate to use if one wants to have the nonlocal model converge to the classical solution as the horizon goes to zero, while keeping the number of nodes inside the horizon the same. The one-point Gauss quadrature is the preferred method for discretizing PD models because it leads to a meshfree model, very well suited for capturing the evolution of damage (or other types of discontinuities) in a material (see e.g. [8, 20, 22, 23]).

The paper is organized as follows: in section 2, we use a constructive approach to derive a PD formulation for 3D dynamic elasticity. In sections 3 and 4, we define the PD stress for three different kernel types and determine the effect of numerical quadrature on computing the peridynamic stress. In section 5, a 1D dynamic elasticity problem with Dirichlet boundary conditions is studied in terms of convergence to the classical solution in the limit of the nonlocal region size going to zero. In section 6 we eliminate the surface effect by using the fictitious nodes method.

\section{Peridynamic formulation for elasticity from a constructive approach}

In this section, we use a constructive approach to derive a PD formulation for 3D dynamic elasticity. We assume that an elastic body $\Omega$ is composed by superposed, uniform imaginary elastic rods (Figure 2A) connecting material points in the domain. Consider a material point $\boldsymbol{x}$, all of the material points connecting with this point forms the nonlocal region $H_{x}$. Figure $2 \mathrm{~A}$ shows the nonlocal region when it is uniform in all directions. If we assume all of the elastic rod can deform only by stretching or compressing and the forces on the ends of the rod are in the direction of the rod, we have a pair-wise model (or bond-based PD model). Consider an imaginary cylinder between points $\boldsymbol{x}$ and $\widehat{\boldsymbol{x}}$ as in Figure 2A, with the area of the end surface 
equal to $A$ and initial length (before loading) $=\|\widehat{\boldsymbol{x}}-\boldsymbol{x}\| . f_{\boldsymbol{x}}$ and $f_{\widehat{x}}$ are forces loaded at the end surfaces. The displacements of the points $\boldsymbol{x}$ and $\widehat{\boldsymbol{x}}$ are, $\boldsymbol{u}(\boldsymbol{x})$ and $\boldsymbol{u}(\widehat{\boldsymbol{x}})$, respectively. Thus, the current position coordinates of the points are $\boldsymbol{y}=\boldsymbol{x}+\boldsymbol{u}(\boldsymbol{x})$ and $\widehat{\boldsymbol{y}}=\widehat{\boldsymbol{x}}+\boldsymbol{u}(\widehat{\boldsymbol{x}})$. We assume no force on the cylinder's side surface. The net force on this cylinder is $f_{\hat{x}}-f_{x}$, and the force direction is along the cylinder length direction after deformation: $\overrightarrow{\boldsymbol{n}}=[\widehat{\boldsymbol{y}}-\boldsymbol{y}] /\|\widehat{\boldsymbol{y}}-\boldsymbol{y}\|$. This net force, on the other hand, is induced by the elastic deformation of the cylinder $(\|\widehat{\boldsymbol{y}}-\boldsymbol{y}\|-$ $\|\widehat{x}-\boldsymbol{x}\|)$, so according to Hooke's Law we have:

$\frac{1}{A}\left(f_{\widehat{\boldsymbol{x}}}-f_{\boldsymbol{x}}\right) \overrightarrow{\boldsymbol{n}}=C(\boldsymbol{x}, \widehat{\boldsymbol{x}}) \frac{\|\hat{\boldsymbol{y}}-\boldsymbol{y}\|-\|\widehat{\boldsymbol{x}}-\boldsymbol{x}\|}{\|\widehat{\boldsymbol{x}}-\boldsymbol{x}\|} \overrightarrow{\boldsymbol{n}}$,

where $\boldsymbol{u}(\boldsymbol{x})$ and $\boldsymbol{u}(\widehat{\boldsymbol{x}})$ are the displacement at the end surfaces, $C(\boldsymbol{x}, \widehat{\boldsymbol{x}})$ is an elastic modulus-like material property. We now define $\boldsymbol{u}_{a}$ as the average displacement for points along this cylinder. According to Newton's second law and Eq. (3), we get:

$\rho_{a} A\|\widehat{\boldsymbol{x}}-\boldsymbol{x}\| \frac{\partial^{2} \boldsymbol{u}_{a}}{\partial t^{2}}=C(\boldsymbol{x}, \widehat{\boldsymbol{x}}) \frac{\|\hat{\boldsymbol{y}}-\boldsymbol{y}\|-\|\widehat{\boldsymbol{x}}-\boldsymbol{x}\|}{\|\widehat{\boldsymbol{x}}-\boldsymbol{x}\|} \overrightarrow{\boldsymbol{n}} A$

where $\rho_{a}=\frac{\rho_{x}}{V_{H_{x}}}$ is the average density in this horizon, is density at $x . V_{H_{x}}$ is the volume of the horizon region for the material point at $\boldsymbol{x}$.
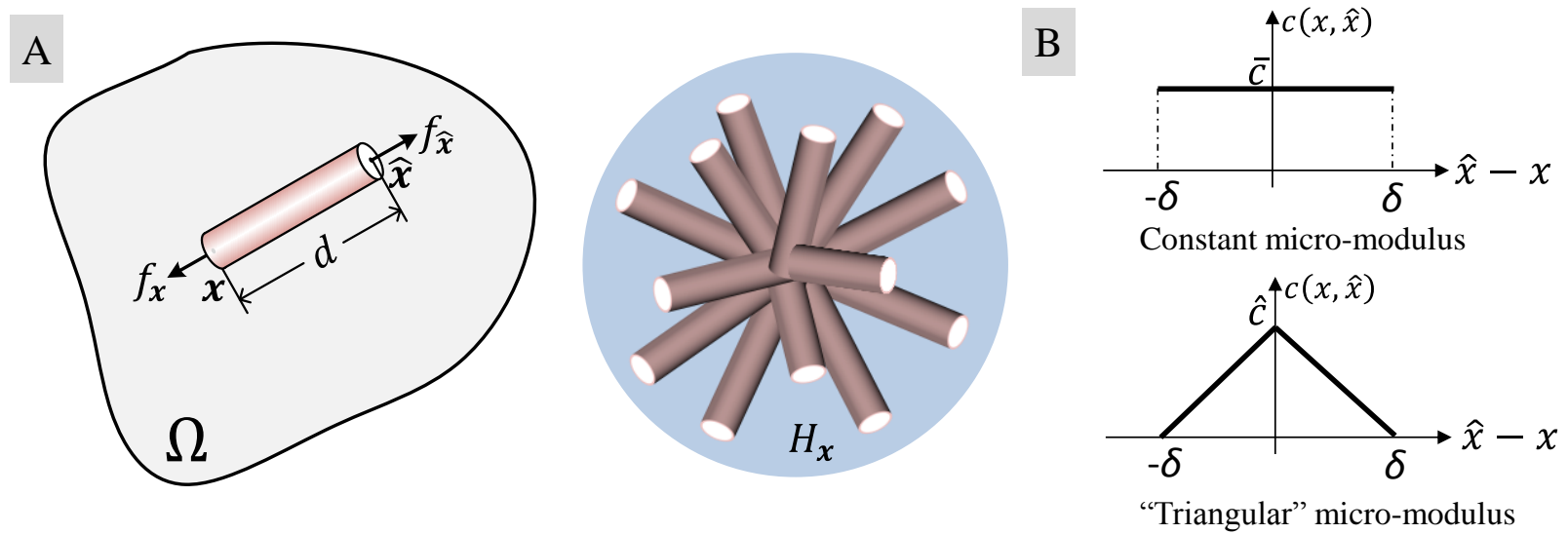

Figure 2. (A) Peridynamic constructive model: (left) material points are connected by imaginary elastic rods and the material is composed of superposed elastic rods; (right) all of the material points connecting with point $\boldsymbol{x}$ form the nonlocal region of this point, $H_{x}$. (B) Two sample functions for the micro-modulus variation inside the PD horizon.

This is where we depart from the classical approach, which assumes the distance $d$ between $\boldsymbol{x}$ and $\widehat{x}$ goes to zero and the relationship above becomes an equation written in terms of the spatial gradient of the displacement field (see e.g. [24]). Here and below, for simplicity, we consider the density to be constant. The case of variable density is an immediate extension of the 
formulation presented here. Dividing Eq. (4) by $A\|\widehat{x}-x\|$ and integrating over the horizon of point $\boldsymbol{x}$ (see Figure 2B) we get:

$\int_{H_{x}} \rho_{a} \frac{\partial^{2} \boldsymbol{u}_{a}(\boldsymbol{x}, \widehat{\boldsymbol{x}}, t)}{\partial t^{2}} \mathrm{~d} V_{\widehat{x}}=\int_{H_{x}} C(\boldsymbol{x}, \widehat{\boldsymbol{x}}) \frac{\|\hat{\boldsymbol{y}}-\boldsymbol{y}\|-\|\widehat{\boldsymbol{x}}-\boldsymbol{x}\|}{\|\widehat{\boldsymbol{x}}-\boldsymbol{x}\|^{2}} \overrightarrow{\boldsymbol{n}} \mathrm{d} V_{\widehat{x}}$

We assume the following relation between the displacement at point $\boldsymbol{x}$ (the center of mass in horizon $H_{x}$ ) at time $t$ and the average displacements in all the mechanical-bonds connected at $\boldsymbol{x}$ :

$\int_{H_{x}} \rho_{a} \frac{\partial^{2} \boldsymbol{u}_{a}(\boldsymbol{x}, \widehat{\boldsymbol{x}}, t)}{\partial t^{2}} \mathrm{~d} V_{\hat{x}}=\rho(\boldsymbol{x}, t) \frac{\partial^{2} \boldsymbol{u}(\boldsymbol{x}, t)}{\partial t^{2}}$

Eq. (5) now becomes:

$\rho \frac{\partial^{2} \boldsymbol{u}(\boldsymbol{x}, t)}{\partial t^{2}}=\int_{H_{x}} c(\boldsymbol{x}, \widehat{\boldsymbol{x}}) \frac{\|\hat{\boldsymbol{y}}-\boldsymbol{y}\|-\|\widehat{\boldsymbol{x}}-\boldsymbol{x}\|}{\|\widehat{\boldsymbol{x}}-\boldsymbol{x}\|^{2}} \overrightarrow{\boldsymbol{n}} \mathrm{d} V_{\widehat{x}}$

with $c(\boldsymbol{x}, \widehat{\boldsymbol{x}})=C(\boldsymbol{x}, \widehat{\boldsymbol{x}})$ defined to be the micro-modulus of the mechanical bond $(\boldsymbol{x}, \widehat{\boldsymbol{x}})$. The micro-modulus function can be very general, while satisfying the restriction pointed out in [25] that the wave speeds are real. Equation (7) is the PD equation for dynamic elasticity, without any body forces. This is a special case of Eqs. (1) and (2), with $n=2$. The formulation is valid for 1D, 2D \& 3D, and body forces can be easily added to this formulation. For 1D model, Eq. (7) reduces to Eqs. (1) and (2) with $n=2$.

In the following sections, we define the PD stress, and then we follow the same procedure as in [11] to perform convergence studies for three different $n$ values ( $n=0,1$, and 2) in Eq. (2), each selected with two kinds of micro-modulus, a constant one and a linearly varying one, on a 1D elastic problem with Dirichlet boundary conditions. Note that larger values of $n$ lead to models dominated by local and nearly-local interactions. Similar statement has been made in [17]. Therefore, for 2D and 3D models, large values of $n$ lead to high dependence of the solution on the discretizing grid. The process in this paper can be directly used to solve problems with Neumann boundary conditions. The PD implementation of Neumann boundary conditions can be seen in [14]. To distinguish between different cases, we will denote those using a constant micromodulus (see Fig. 2B) by C1, C2, C3 for $n=0,1$, and 2, respectively, and those for the linear (also called "triangular" in [14], see Fig. 2B) by L1, L2, and L3 for $n=0$, 1, and 2, respectively. C3 and L3 are cases corresponding to the kernel derived above.

\section{Peridynamic stress definition}

In this section, we directly apply the PD force flux definition given by Lehoucq and Silling [26] and simplify it to define the PD stress in 1D: force through a given surface at a certain geometrical point (See Figure 3A). According to this definition, we have the PD stress:

$\sigma_{p}(x, t)=\int_{x}^{\delta+\tilde{x}} \int_{x-\delta}^{x} f_{n}(\tilde{x}, \hat{x}, t) \mathrm{d} \tilde{x} \mathrm{~d} \hat{x}$.

We could also define the PD stress as the rate at which force is transferred through a material point across a given surface at that point which is similar to the definition of heat flux in [14] 
(see Figure 3C). In [11], we showed that the two definitions for PD heat flux are equivalent. The proof for the case of stress is identical, and it will not be repeated here. For the results shown in the next sections, we use Lehoucq and Silling's definition of stress. Utilizing the other definition leads to identical results.

\section{A}

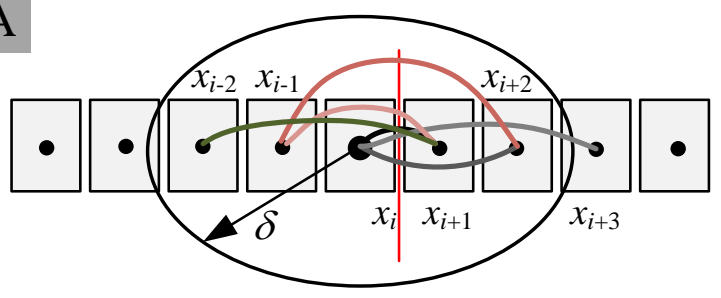

C

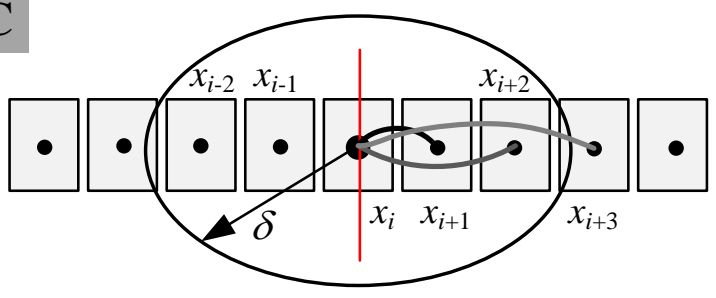

B

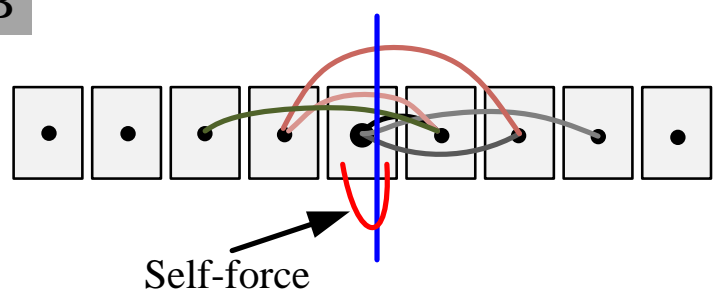

$\mathrm{D}$

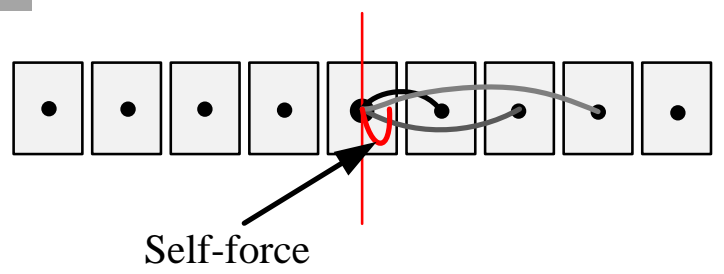

Figure 3. Definitions for peridynamic stress: (A) the force/unit area through a given surface at a certain geometrical point; (B) when the surface "cuts through" a node, a "self-force" (red curve) has to be included in computing the PD stress with the definition in (A); (C) the force/unit-area transferred through a material point across a given surface at that point; (D) the self-force that has to be included in computing PD stress with the definition in (C).

The specific micro-modulus function $c(x, \hat{x})$ can be determined if we assume a certain profile and equate the PD stress to the classical stress arising from a simple linear displacement profile under static conditions. Let the imposed displacement field on an 1D infinite elastic bar be $a x+b$, with $a$ and $b$ given constants. The classical stress at any point along the bar is:

$$
\sigma=E \frac{\partial(a x+b)}{\partial x}=E a,
$$

where $E$ is the elastic modulus. Substituting this displacement field into Eqs. (2) and (8), yields:

$\sigma_{p}^{(n)}(x)=\int_{x}^{\delta+\tilde{x}} \int_{x-\delta}^{x} c(|\hat{x}-\tilde{x}|) \frac{a}{|\hat{x}-\tilde{x}|^{n-1}} \mathrm{~d} \tilde{x} \mathrm{~d} \hat{x}$

Two profiles for the micro-modulus function are considered. One is a constant independent of the bond length (see Figure 1 and 2B):

$c(|\hat{x}-x|)=\bar{c}$

another one varies linearly over the horizon size ( called "triangular" micro-modulus):

$c(|\hat{x}-x|)=\hat{c}\left(1-\frac{|\hat{x}-x|}{\delta}\right)$. 
With this micro-modulus, shorter bonds have a "higher" influence compared with the longer bonds in the elastic response at point $x$. Substituting (11) and (12) into (10), we obtain the PD stress at any point $x$ along the bar:

$\sigma_{p}^{(n)}=\frac{\delta^{(3-n)}}{(3-n)} \bar{c} a$,

when $c(|\hat{x}-x|)=\bar{c}$, and

$\sigma_{p}^{(n)}=\frac{\delta^{(3-n)}}{(4-n)(3-n)} \hat{c} a$,

when $c(|\hat{x}-x|)=\hat{c}\left(1-\frac{|\hat{x}-x|}{\delta}\right)$. The parameter $n$ is the exponent in Eq. (2). Equating (10), (11) with the classical stress in (9), gives:

$\bar{c}(n)=\frac{(3-n) E}{\delta^{(3-n)}}$

$\hat{c}(n)=\frac{(4-n)(3-n) E}{\delta^{(3-n)}}$.

In the next section, we introduce the numerical discretization for evaluating the PD stress and analyze the effect the relative grid density factor, the value $m=\frac{\delta}{\Delta x}$, with $\Delta x$ being the uniform spatial discretization size, has on the solution.

\section{The effect of spatial quadrature on computing the peridynamic stress}

In this section, we determine the effect of numerical quadrature on computing the PD stress for the six types of kernels mentioned above. For a certain horizon size $\delta$ we select a uniform grid to discretize the domain with grid spacing $\Delta x$. The goal is to observe the error (the difference from the classical local solution) introduced by numerical quadrature for different choices of the kernel function, for different "relative grid density factors" $m=\frac{\delta}{\Delta x}$, and for different horizon sizes. Non-uniform grids are possible in discretizing the peridynamic equations (see e.g. [15, 27, 28]), but this is not pursued here. Note that when using non-uniform grids with midpoint quadrature, only the weak patch test will be passed under appropriate convergence analysis, and extreme care should be taken. To compute the integral over $H_{x}$ in Eq. (1) we use the one-point Gauss quadrature (the mid-point integration) scheme. Each node has a "volume", in $1 \mathrm{D}$ given by the length of the segment associated with it, in our case $\Delta x$. The nodes at the ends have the coordinates $\Delta x / 2$ and $L-\Delta x / 2$ for the bar of length $L$. Other options can be selected: for example, we can place nodes at the actual ends of the bar in which case these end nodes will have half-volumes (see [12]). The results we report below use the first method, with all nodes having the same volume (length).

The micro-force at a node $x_{i}$ due to the bond with the node $x_{p}$ inside its horizon is: 
$f\left(x_{p}, x_{i}\right)=c_{n}\left(\left|x_{p}-x_{i}\right|\right) \frac{u\left(x_{p}, t\right)-u\left(x_{i}, t\right)}{\left|x_{p}-x_{i}\right|^{n}}$,

and this leads to the following spatial discretization of the PD equation:

$\rho \frac{\partial^{2} \boldsymbol{u}\left(x_{i}, t\right)}{\partial t^{2}}=\sum_{p} c_{n}\left(\left|x_{p}-x_{i}\right|\right) \frac{u\left(x_{p}, t\right)-u\left(x_{i}, t\right)}{\left|x_{p}-x_{i}\right|^{n}} V_{p}$,

where the summation is after all nodes $x_{p}$ inside the horizon of node $x_{i}$, and $V_{p}$ is the portion of the volume of the node $x_{p}$ actually covered by the horizon of node $x_{i}$.

To investigate the error introduced by the mid-point integration for each of the kernels considered in section 3, we use the discretized forms in (17) and (18) for a point away from the ends of the bar to calculate the peridynamic stress at $x_{i}$ for the linear displacement field $a x+b$. According to Figure 3A, we obtain:

$\sigma_{p}^{(n)}\left(x_{i}\right)=\sum_{i, p} c\left(\left|x_{p}-x_{i}\right|\right) \frac{a}{\left|x_{p}-x_{i}\right|^{n-1}} V_{p} V_{i}$

where $x_{p}, x_{i}$ are in the same family and $1 \leq \frac{x_{p}-x_{i}}{\Delta x}<m+\frac{1}{2}$. Note that $m=\frac{\delta}{\Delta x}$ is the relative grid density factor mentioned before. With (15) and (16), combining (19) and (9) yields:

$\frac{\sigma_{p}^{(n)}}{\sigma}=\left\{\begin{array}{l}\frac{3-n}{m^{(3-n)}}\left[-\frac{1}{2} m^{(2-n)}+\sum_{j=1}^{m} j^{(2-n)}\right], \text { for the constant micromodulus } \\ \frac{(4-n)(3-n)}{m^{(4-n)}} \sum_{j=1}^{m-1}(m-j)^{(2-n)} j, \text { for the triangular micromodulus }\end{array}\right.$

The total force exerted on a material point is the divergence of the stress. By analyzing the PD stresses for different choices of $n$ and different discretizations, and comparing them with the stress from the local model, we can observe the dependence of the PD solution on the parameters $n$ (used in the kernel) and $m$ (the horizon factor used in the discretization). In Eq. (19), we lack the "self-force" term (Figure 3B and 3D). We compute the "self-force" term (from the right side of the node's position) by approximating it with (see [14]):

$\frac{1}{2}\left[c_{n}\left(\left|x_{i+1}-x_{i}\right|\right) \frac{u\left(x_{i+1}, t\right)-u\left(x_{i}, t\right)}{\left|x_{i+1}-x_{i}\right|^{n}}+c_{n}\left(\left|x_{i-1}-x_{i}\right|\right) \frac{u\left(x_{i-1}, t\right)-u\left(x_{i}, t\right)}{\left|x_{i-1}-x_{i}\right|^{n}}\right]$.

This term represents the force exerted by the left half onto the right half of the nodal volume across a mathematical surface passing through the node (center of the volume in the one-point Gaussian quadrature) (see [11]). If we do not add this term, the stress calculated will be slightly smaller. The error of not including this stress is amplified when a larger $n$ value is used because the region near the node has a higher influence on the total integral when $n$ is larger. Adding this term into Eq. (19), yields:

$\frac{\sigma_{p}^{(n)}}{\sigma}=\left\{\begin{array}{c}\frac{3-n}{m^{(3-n)}}\left[\frac{1}{2}-\frac{1}{2} m^{(2-n)}+\sum_{j=1}^{m} j^{(2-n)}\right], \text { for the constant micromodulus } \\ \frac{(4-n)(3-n)}{m^{(4-n)}}\left[\frac{m}{2}+\sum_{j=1}^{m-1}(m-j)^{(2-n)} j\right], \text { for the triangular micromodulus }\end{array}\right.$ 
In particular, when $n=0$,

$\frac{\sigma_{p}^{(0)}}{\sigma}=\left\{\begin{array}{c}1+\frac{1}{2 m^{2}}+\frac{3}{2 m^{3}},(\text { case C1) } \\ 1-\frac{1}{m^{2}}+\frac{6}{m^{3}},(\text { case L1) }\end{array}\right.$.

When $n=1$,

$\frac{\sigma_{p}^{(1)}}{\sigma}=\left\{\begin{array}{l}1+\frac{1}{m^{2}},(\text { case C2) } \\ 1+\frac{2}{m^{2}},(\text { case L2) }\end{array}\right.$.

When $n=2$,

$\frac{\sigma_{p}^{(2)}}{\sigma}= \begin{cases}1, & (\text { case C3) } \\ 1, & (\text { case L3) }\end{cases}$

Note that $m$ does not have to be an integer. The mid-point integration method is exact (meaning it gives the same value of PD stress as the classical stress for this uniform deformation) only in the cases C3 and L3. In all other cases ( $n=0$ and $n=1$ with the constant or linear micromodulus), the numerical error goes to zero as $m$ goes to infinity (the number of nodes inside the horizon increases) but it is never zero for a given $m$-value. Notice also that for the kernels generated by $n=0$ and $n=1$, when $m$ is small, the integration error is rather significant. This error is independent of the horizon size, implying that for a fixed and small value $m$, the peridynamic stress based on kernels generated by $n=0$ and 1 converges, as the horizon goes to zero, to a different value than the expected classical one for the given linear displacement. In conclusion, for constant or linear micro-modulus, one-point Gauss quadrature (mid-point integration) produces the exact, classical stress for a homogeneous deformation only for the kernel with $n=2$, while with the other two kernel types, this quadrature converges to the exact value only in the limit of the number of nodes inside the horizon going to infinity. The same behavior was observed in the diffusion problem modeled by PD (see [11]).

With this understanding of the connection between the kernel type and numerical quadrature of the peridynamic stress, in the next section we study the convergence properties of the peridynamic solution to the classical solution, in the limit of the horizon size going to zero. Note that with approximating the inclusion of the nodal self-force from Eq. (21) into Eq. (18), the discretized form (in space) of the peridynamic elasticity becomes:

$\rho \frac{\partial^{2} \boldsymbol{u}\left(x_{i}, t\right)}{\partial t^{2}}=$

$\sum_{p} c_{n}\left(\left|x_{p}-x_{i}\right|\right) \frac{u\left(x_{p}, t\right)-u\left(x_{i}, t\right)}{\left|x_{p}-x_{i}\right|^{n}} V_{p}+$

$\frac{1}{2}\left[c_{n}\left(\left|x_{i+1}-x_{i}\right|\right) \frac{u\left(x_{i+1}, t\right)-u\left(x_{i}, t\right)}{\left|x_{i+1}-x_{i}\right|^{n}}+c_{n}\left(\left|x_{i-1}-x_{i}\right|\right) \frac{u\left(x_{i-1}, t\right)-u\left(x_{i}, t\right)}{\left|x_{i-1}-x_{i}\right|^{n}}\right]$

(26) 
Note also that for any real number $n$ smaller than or equal to 2, Eq. (22) converge to 1 when $m$ goes to infinity, which implies that other kernel types may be considered.

\section{Convergence of peridynamic results for different kernels to the classical, local model}

In this section, we analyze convergence of the peridynamic model to the classical solution of an elastic wave propagation problem. Here, we consider a Gaussian wave propagating along a 1D bar with fixed ends. Similar example with free ends in peridynamics is studied in [12] where the kernel given by $n=1$ was used. The length of the bar is $L=1 \mathrm{~m}$, Young's modulus $E=200$ GPa, density $\rho=8000 \mathrm{~m}^{3} / \mathrm{s}$. The initial displacement imposed on the elastic bar is a Gaussian of the form $u(x, 0)=0.02 \exp \left(-100(x-0.5)^{2}\right) \mathrm{m}$, and is shown in Figure $4(t=0 \mathrm{~ms})$. The initial velocity of the bar $v(x, 0)=0$. The classical formulation for this problem is as follows:

$\frac{\partial^{2} u(x, t)}{\partial t^{2}}=\frac{E}{\rho} \frac{\partial^{2} u(x, t)}{\partial x^{2}}, 0<x<L, 0 \leq t<\infty$,

$u(x, 0)=0.02 \exp \left(-100(x-0.5)^{2}\right), 0<x<L$,

$v(x, 0)=0,0<x<L$,

$u(0, t)=u(L, t)=0,0 \leq t<\infty$,

Using the traveling wave method, we can solve the above problem exactly.

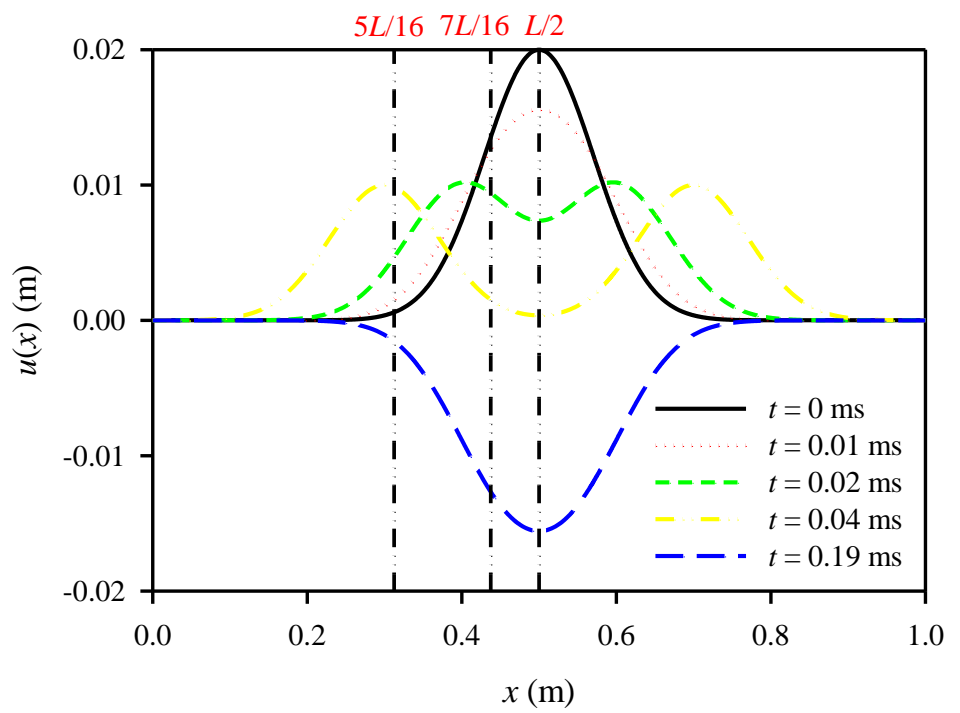

Figure 4. The classical solution for the displacement at various times for an elastic wave in a 1D bar.

The classical solution is plotted, at a few time instances, in Figure 4. The displacements at times $0.01 \mathrm{~ms}, 0.02 \mathrm{~ms}$ and $0.19 \mathrm{~ms}$ at location $7 L / 16$ (see Fig. 4), are used to evaluate 
convergence of the PD solution to the classical solution in the limit of the horizon going to zero. For time integration in PD, we use the fourth-order Runge-Kutta method, while for the spatial integration the midpoint rule is used. We select a time step $\Delta t=0.1 \mathrm{~ns}$ in all tests as this is a stable time step for all of the cases studied here. The PD solutions with the three kernel types and the two different micro-modulus are shown in Figure 5 (at $t=0.01 \mathrm{~ms}$ ), Figure 6 (at $t=0.02 \mathrm{~ms}$ ) and Figure 7 (at $t=0.19 \mathrm{~ms}$ ).

Here, the Dirichlet boundary conditions are implemented by keeping the displacement at the end nodes equal to zero at all times. As each node has a certain length in the 1-D case, this implementation of boundary conditions is only approximate; as the mesh is refined, e.g. $m \rightarrow \infty$, the region over which displacement is set to zero converges to the end points of the bar. Therefore, this implementation of the Dirichlet boundary conditions converges, in the limit of $m \rightarrow \infty$, to the classical boundary condition. Note that because node locations vary for differing horizon sizes and grid-densities, obtaining the displacement at a specified location requires linear interpolation of values calculated at adjacent nodes. The reason for using this approximate implementation of boundary conditions is that it is the simplest one and similar to how boundary conditions are implemented in, for example, the FEM of the local problem. In section 6, we will investigate the behavior of the nonlocal solution when the boundary condition is implemented exactly. 

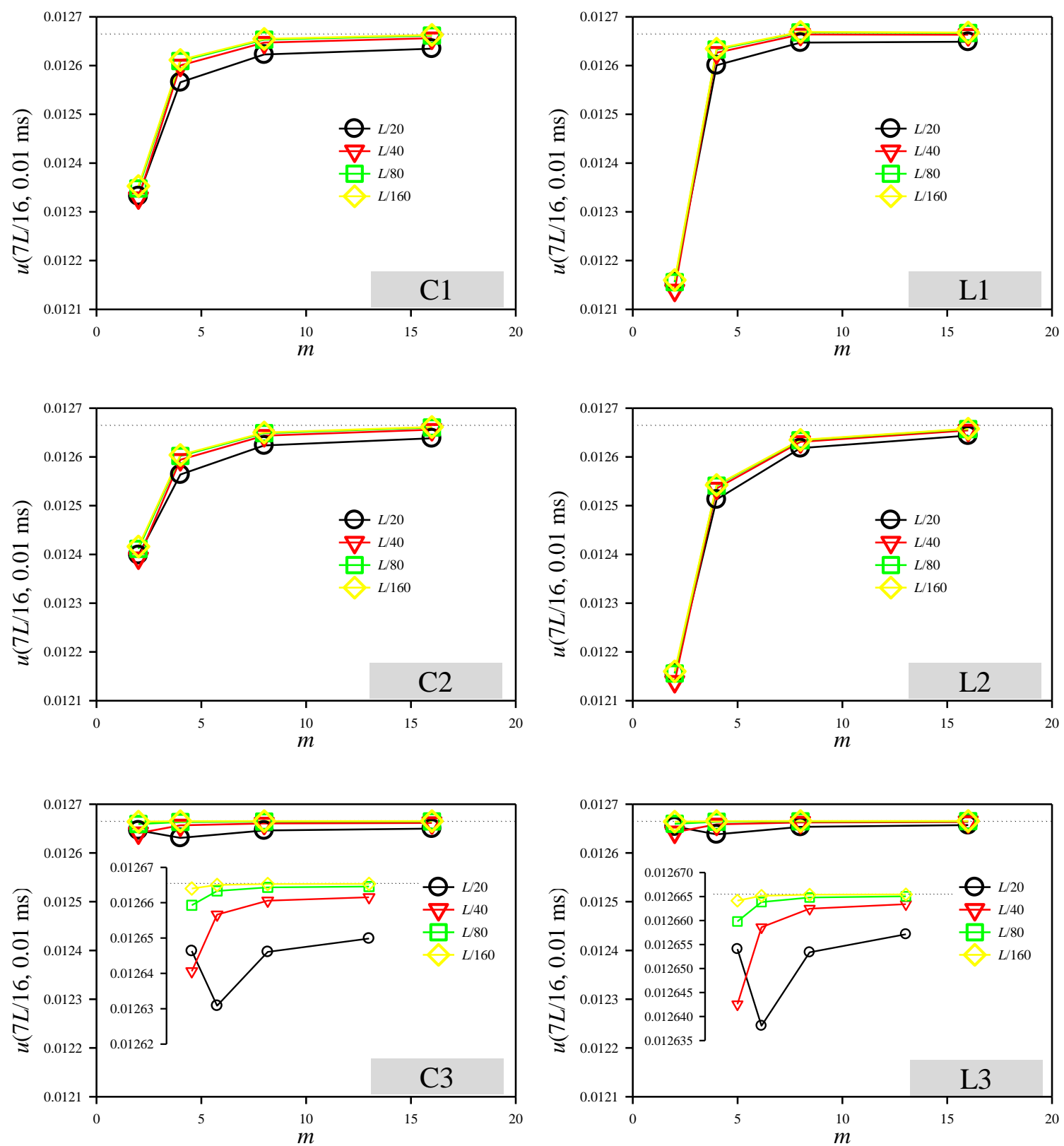

Figure 5. The $m$-convergence curves for four different horizon sizes ( $L$ divided by 20, 40, 80, and $160)$ with approximate boundary conditions. The displacement at $x=7 L / 16$ and $t=0.01 \mathrm{~ms}$ for different values of $m$ and different horizon sizes. The dotted line indicates the value of the classical exact displacement at the same location and time. For C3 \& L3 we include inserts that zoom in the ordinate axis to observe the behavior easier. 

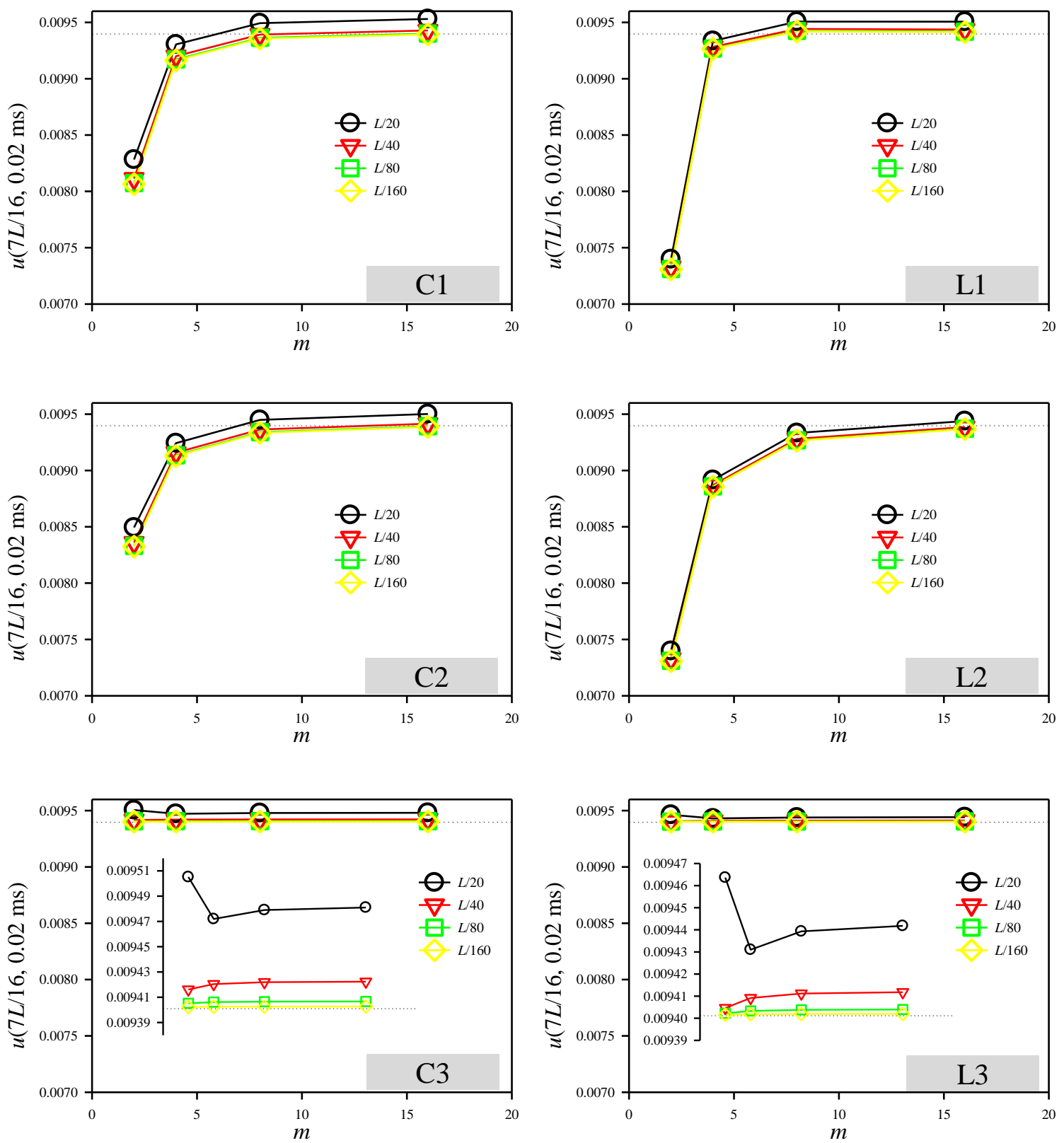

Figure 6. Solutions, using approximate boundary conditions, for displacements at $x=7 L / 16$ and $t=0.02 \mathrm{~ms}$ for different values of $m$ and different horizon sizes. The dotted line indicates the value of the classical exact displacement at the same location and time. For C3 and L3 we include inserts that zoom in to observe the behavior easier. 

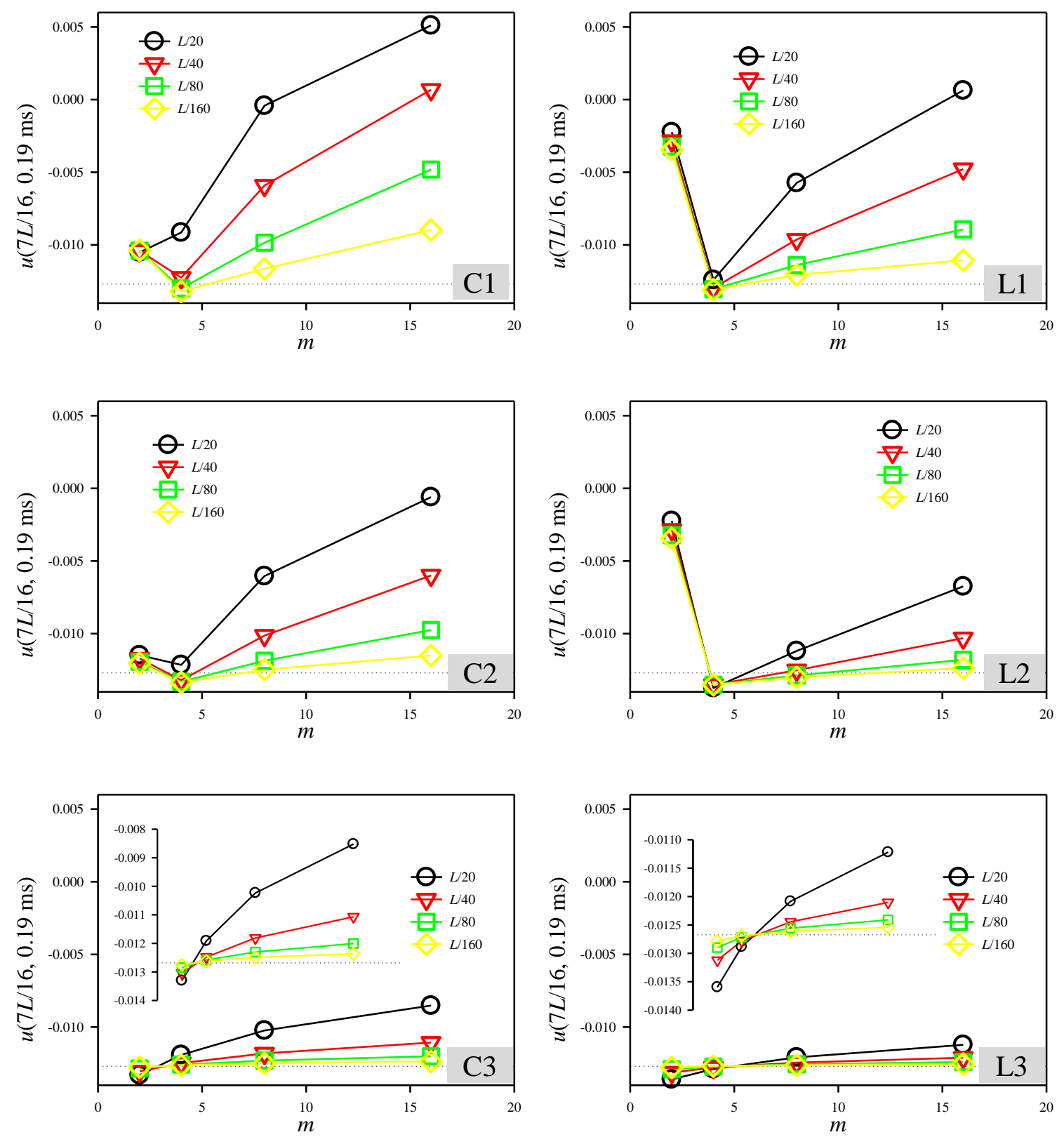

Figure 7. Displacements, using approximate boundary conditions, at $x=7 L / 16$ and $t=0.19 \mathrm{~ms}$ plotted for different values of $m$ and different horizon sizes. The dotted line indicates the value of the classical exact displacement at the same location and time. The zoom-ins for cases C3 and L3 show clearly that the $m$-convergence curves are all crossing at the classical solution for $\mathrm{C} 3$ and L3. This was first reported in [14], and the behavior is explained in detail in [11].

Similar to what was done for the diffusion problem in [11], we study $m$-convergence and $\delta$ convergence (see [12]) for this elastic wave propagation problem. In the $m$-convergence we consider the horizon $\delta$ fixed and take $m \rightarrow \infty$. The numerical PD approximation will converge to the exact nonlocal PD solution for the given $\delta$. In the $\delta$-convergence, the horizon $\delta \rightarrow 0$ while 
$m$ is fixed or increases with decreasing $\delta$. For $\delta$-convergence and in problems with no singularities, the numerical PD solutions are expected to converge to the classical local solution.

The results shown in Figures 5, 6 and 7 support expectations and previous findings regarding the exactness of one-point Gauss quadrature on constant and linear micro-modulus. For example, the kernel with $n=2$ (cases C3 and L3) exhibits $\delta$-convergence to the classical solution for any fixed value of $m$ as $\delta \rightarrow 0$. This happens because of the exact nature of the numerical quadrature: $\frac{\sigma_{p}^{(2)}}{\sigma}=1$, for any $m$ (see Section 4). A similar observation has been made in $[29,30]$ in a slightly different context and for the steady-state case, the focus there being on determining the quadrature method that renders a particular numerical scheme assymptotically compatible, independent of the kernel type. These authors found that a certain finite difference discretization allows a very general choice of the kernel. Our interest here is, however, in the one-point Gaussian quadrature method since this is the one that leads to practical, meshfree, discretizations for fracture and failure problems, in which peridynamics shows its greatest advantages. For kernels produced by $n=0$, and $n=1$, the peridynamic numerical solution (when one-point Gauss quadrature is used for the spatial integration) converges, but to a wrong value if $m$ is kept fixed and the horizon goes to zero (see Figures 5, 6 and 7). Therefore, the main difference between $n=2$ and $n=0$ or 1 , in terms of $\delta$-convergence behavior, is not the convergence rate, but the convergence limit. We note that, as $m$ increases, the "wrong" limit points approach the correct limit, as the horizon goes to zero. In other words, with $\delta \rightarrow 0$ and $m \rightarrow \infty,(\delta m$-convergence in [12]) then all of the cases converge to the classical solution. Therefore, in terms of this type of convergence, all of these kernel functions are valid. Similar conclusion has also been drawn by [29] for the steady-state case.

The convergence results in Figure 7 reveal a pitfall in performing computational experiments with nonlocal models and comparing them with local solutions. One can easily notice the excellent "match" of the nonlocal solution to the classical solution, for a certain horizon size and a certain discretization (for some small $m$-values), and declare that "validation" of the nonlocal model has been achieved. This pitfall has been explained in [11] for the diffusion case. When one selects a finer discretization, with the fixed horizon size, strangely enough, the nonlocal solution starts to depart from the local, classical solution, even though one uses a more accurate quadrature with a finer discretization grid! This type of behavior is likely what happens with the excellent, but unexplained, match between peridynamic results and classical results shown in, for example, [13], for one horizon size and one particular grid spacing. The lesson to be learned is that agreement of a PD result with the local model does not necessarily imply that the PD discretization is a converged solution. Special care needs to be taken when nonlocal models are discretized.

The above convergence behaviors are similar to that for the peridynamic diffusion model in [11]. Table 1 shows the comparison between dynamic elasticity and transient diffusion PD solution in terms of convergence behavior for the case of implementing Dirichlet boundary conditions approximately, as explained above. In diffusion, the infinite heat "propagation speed" makes the effect of boundary conditions to be felt instantly at every point in the material, while 
in dynamic elasticity (with a finite wave propagation speed), the way boundary conditions are imposed affects the displacement evolution non-uniformly in time. Therefore, in dynamic elasticity, the solution is not always affected by the way boundary conditions are imposed. For example, before the wave bounces off the fixed ends $(t=0.01,0.02 \mathrm{~ms}$, see Figures 5 and 6$)$, the solutions are not affected by boundary conditions, while the solutions "feel" the presence of the boundary conditions after the wave bounces $(t=0.19 \mathrm{~ms}$, see Figure 7$)$.

Remark. Note that in Table 1, for transient diffusion, all $m$-convergence curves cross at a single point for the case of imposing Dirichlet boundary conditions in the approximate way (see [11]). In [11], we attributed the crossing to the competition between the approximate way of implementing the boundary conditions and nonlocal effects. In [11], we showed that when the Dirichlet boundary conditions are imposed exactly (using fictitious nodes, see next section), the curves do not cross but converge assympotically to the nonlocal solution value, and the $\delta$ convergence is monotonical for any $m$-value. See examples in [11, 31]. For elasticity, the crossing of $m$-convergence curves at the same point may happen because of the boundary conditions/nonlocal effect interaction, but this feature tends not to persist in time and is being "destroyed" by the nonlocal wave dispersion. Nonlocal dispersion is also responsible for the $m$ convergence curve of the larger horizon to cross the other curves, while the rest do not cross. For this, please see the discussion below and Figures 5 and 8.

\begin{tabular}{lll}
\hline $\begin{array}{l}n \text { value of the } \\
\text { constructive kernel }\end{array}$ & Dynamic elasticity & Transient diffusion \\
\hline $\begin{array}{l}\text { Convergence } \\
\text { characteristics }\end{array}$ & $\begin{array}{l}\text { All converge (under } \delta m \text { - } \\
\left.\text { convergence }{ }^{1}\right) \text { to the classical } \\
\text { solution. The kernel with } n=2 \\
\text { has fastest convergence. }\end{array}$ & $\begin{array}{l}\text { All converge (under } \delta m \text { - } \\
\text { convergence) to the classical } \\
\text { solution. The kernel with } n=2 \text { has } \\
\text { fastest convergence. }\end{array}$ \\
\hline $\begin{array}{l}\text { "Wave" speed } \\
(n=0,1)\end{array}$ & $\begin{array}{l}\text { Finite } \\
\text { Infinite }\end{array}$ \\
\hline $\begin{array}{l}m \text {-convergence curves } \\
(n=2)\end{array}$ & $\begin{array}{l}\text { May or may not cross. If crossing, } \\
\text { may not be at the same point. }\end{array}$ & $\begin{array}{l}\text { All cross at a single point, which is } \\
\text { not the classical solution. }\end{array}$ \\
& $\begin{array}{l}\text { at the same point, that is also the } \\
\text { classical solution. }\end{array}$ & $\begin{array}{l}\text { All cross at a single point, which is } \\
\text { the classical solution. }\end{array}$ \\
\hline
\end{tabular}

Table 1. Comparison between solution behavior relative to the kernel type for the PD models of dynamic elasticity and transient diffusion. The behavior of the $m$-convergence curves refers only to the case of Dirichlet boundary conditions implemented approximately.

The convergence behavior varies with time and location in the peridynamic elasticity model. For example, at $t=0.01 \mathrm{~ms}$, PD solutions converge to values smaller than the classical

\footnotetext{
${ }^{1}$ See reference [12].
} 
solution (see Figure 5), while at $t=0.02 \mathrm{~ms}$, PD solutions converge to values larger than the classical solution (see Figure 6). To fully understand this feature, we now look into the PD solution at different locations and times (see Figure 8). Since the constructive kernel $(n=2)$ has superior convergence compared with the postulated versions $(n=0,1)$, in Figure 8 we only show case C3. The L3 case has a similar behavior. Different from the diffusion model, the convergence curves in Figure 8 may or may not cross. If they all cross at the same point, that point is also the classical solution value (see Figure 8, row 3, columns 1 and 2). Please see reference [11] for the proof of this for the case of transient diffusion. The behavior of the $m$ convergence curves up to the point at which the presence of the boundary conditions influences the solution is influenced only by the nonlocal dispersion, and that can be seen from the top two rows in Figure 8 for the $m$-convergence curve of the largest horizon size, which has the largest nonlocal dispersion. As time goes on, after the bouncing of the waves from the boundaries, nonlcoal dispersion starts to "destroy" the crossing of the $m$-convergence curves. 

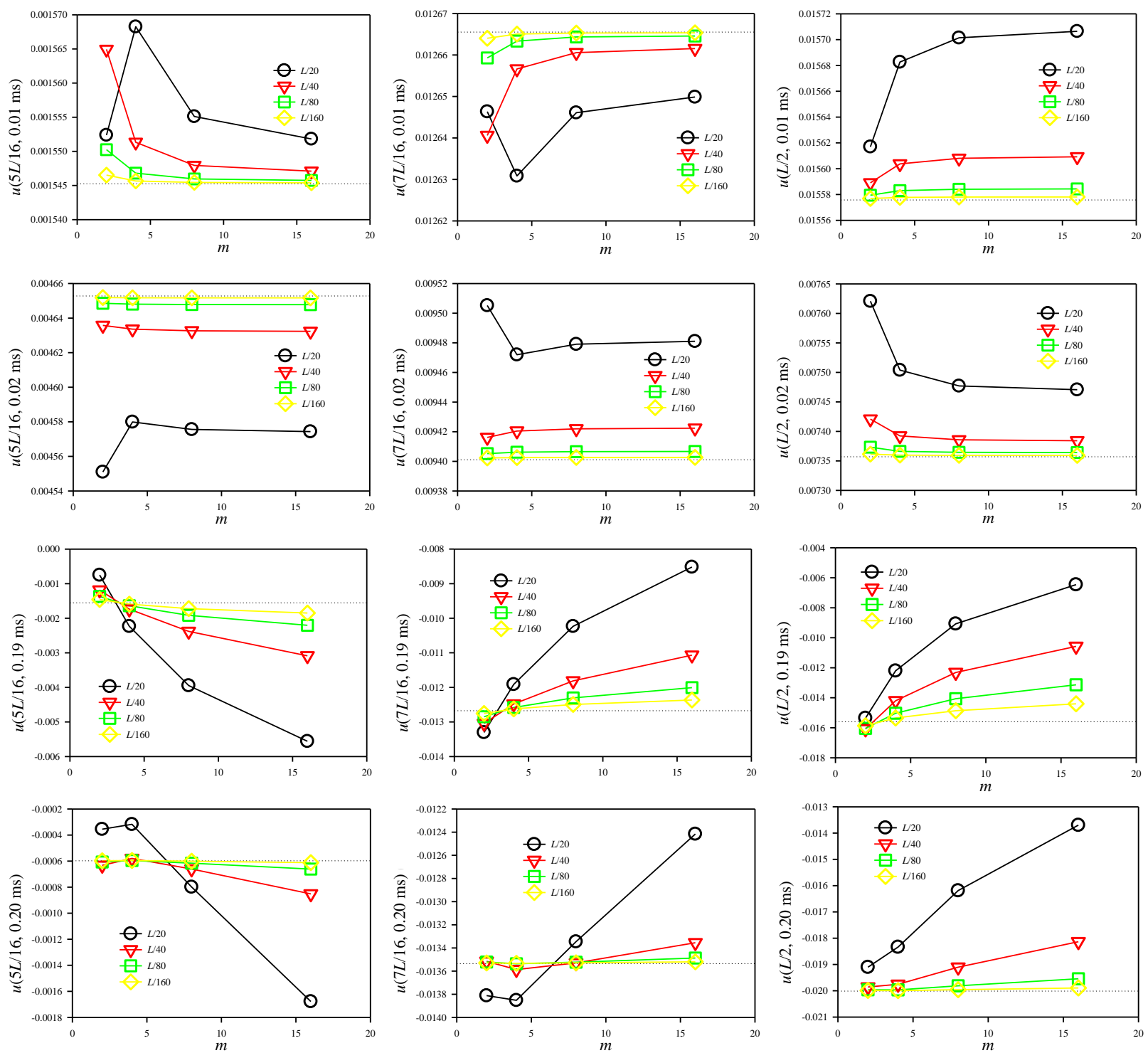

Figure 8. The $m$-convergence curves, using approximate boundary conditions, for kernel $\mathrm{C} 3$ at three different locations: $x=5 L / 16$ (left column), $x=7 L / 16$ (middle column) and $x=L / 2$ (right column), four different times: $t=0.01 \mathrm{~ms}$ (top row), $0.02 \mathrm{~ms}$ ( $2^{\text {nd }}$ row), $0.19 \mathrm{~ms}$ ( $3^{\text {rd }}$ row) and $0.20 \mathrm{~ms}$ (last row). In each panel results are from four different horizon sizes: $L / 20, L / 40, L / 80$ and $L / 160$. The dotted lines represent the values of the classical solution at the corresponding locations and times. 


\section{Fictitious Boundary Method for Applying the Boundary Conditions}

In this section, we apply fictitious boundaries to the ends of the 1D bar (See Figure 9). We use these extra nodes to impose the boundary conditions in an exact way, and also eliminate the surface effect (see [13]). Without the fictitious boundaries, the nodes at the ends of the bar have a smaller "family" (fewer mechanical bonds) than those in the bulk. If one uses the same micromodulus as those produced for the bulk nodes, we end up with a so-called "skin effect" or surface effect (see also [5] for a detailed description). To correct this surface effect, several approaches have been proposed (see a review in [32]). Here we apply the fictitious node method. The addition of the fictitious nodes is trivial in one dimension, however, in 2D or 3D and for complex geometries, fictitious boundaries may become unwieldy to implement and use. Moreover, for problems in which new surfaces are created, the fictitious nodes approach is likely unusable [32].

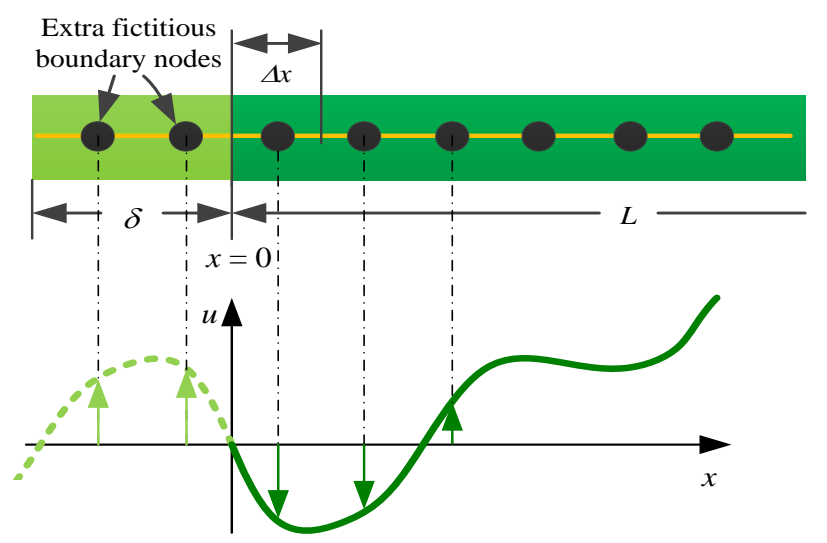

Figure 9. Fictitious nodes are added outside of the domain over a length $\delta$ to eliminate the surface effect (see $[11,13])$. 

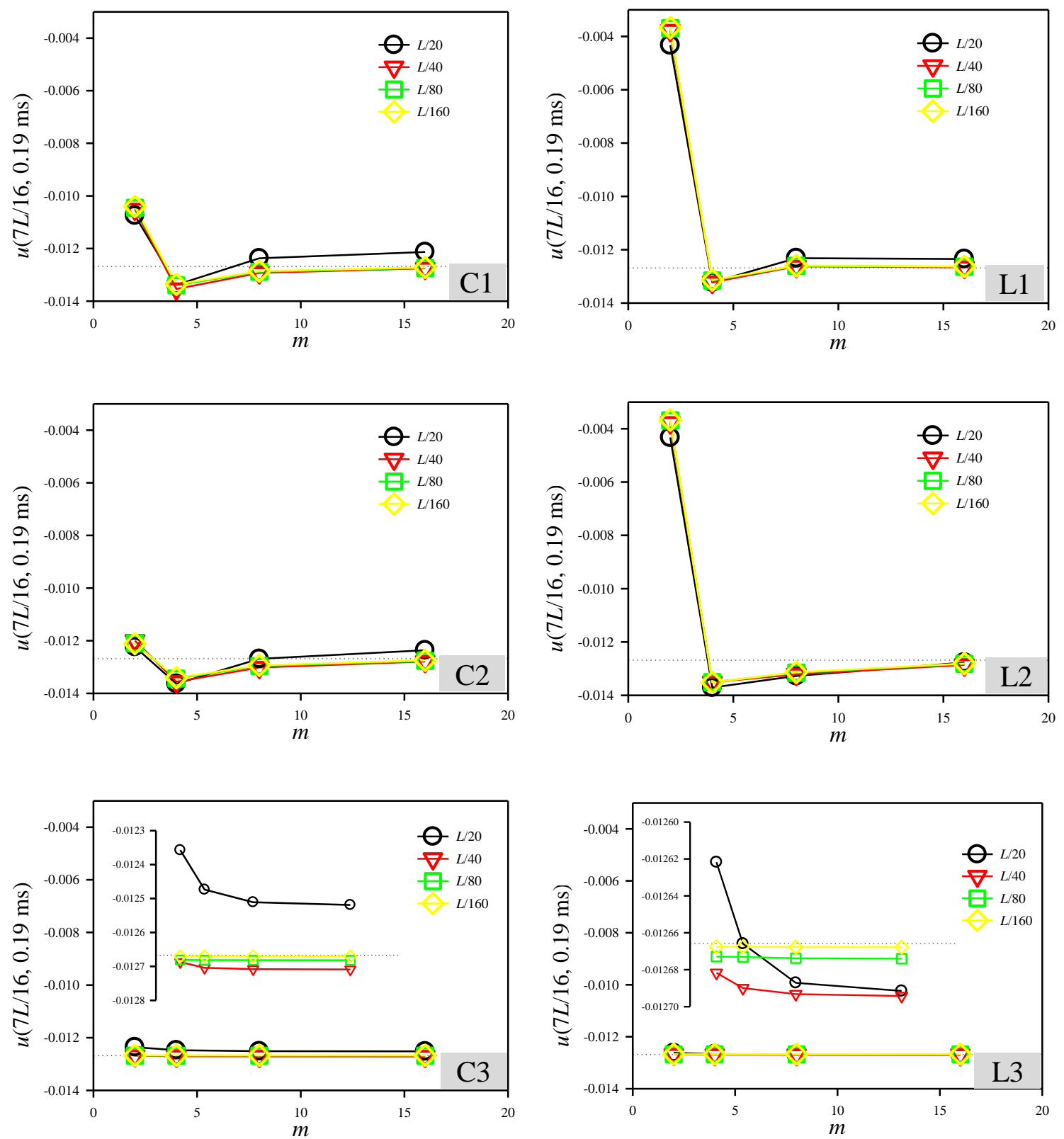

Figure 10. The $m$-convergence curves for four different horizon sizes with fictitious node method for imposing the boundary conditions. The displacement at $x=7 L / 16$ and at $t=0.19 \mathrm{~ms}$ (after bouncing from the ends) is plotted for different values of $m$ and $\delta$. The dotted line indicates the value for the classical exact displacement at the same location and time. In the C3 and L3 cases we include zoom-ins to better distinguish between the nonlocal solutions, which, in these cases, are very close to the classical value.

Figure 10 shows the $m$-convergence curves results for the six different kernels types. Dirichlet boundary conditions are applied on all of the fictitious nodes (see Figure 9 and [13]), and the nodes in the actual bar are free from any conditions. The displacement and velocity 
variations in the fictitious region are the negative mirror image of the values near the boundary in the actual bar as shown in Figure 9. This way of imposing Dirichlet boundary conditions effectively keeps the geometrical ends of the bar fixed, similar to what would happen in a finite element discretization of the classical model. Thus, we can say that the boundary conditions are now implemented exactly. Thus, convergence behavior should only be affected by the numerical integration error and nonlocality, including in the form of nonlocal wave dispersion.

For the kernels of cases C3 and L3, different from the results in Figure 7, in Figure 10 the $m$-convergence curves, except for the one corresponding to the largest horizon size, do not cross at all. These $m$-convergence curves with small horizon sizes $(\delta<L / 20)$ asymptotically converge to the nonlocal solution of their corresponding horizon used. For these curves, we also have monotonic $\delta$-convergence to the classical value, for all $m$-values. The $m$-convergence curve for the larger horizon size $L / 20$ crosses the classical value. The reason for this different behavior is the higher nonlocal wave dispersion introduced by the large nonlocal region.

For all kernel types, as the horizon gets smaller and $m$ grows larger ( $\delta m$-convergence), the peridynamic solutions approach the classical one. Notice, however, that for the kernels with $n=$ 0 or $n=1$, the limit of $\delta$-convergence (fixed $m$ ) is not the classical one. This is an important observation that practitioners of peridynamics should be aware of. We have now shown that $\delta$ convergence to values other than the classical one for kernels with $n \neq 2$ is independent on the way boundary conditions are applied, exactly or approximately.

Another observation by comparing Figure 10 and Figure 7 is that the PD solutions with fictitious nodes have smaller errors than the ones without.

\begin{tabular}{lrrrrrr} 
Relative difference $(\%)$ & $\mathrm{C} 1$ & $\mathrm{C} 2$ & $\mathrm{C} 3$ & $\mathrm{~L} 1$ & $\mathrm{~L} 2$ & $\mathrm{~L} 3$ \\
\hline with fictitious nodes & 1.741 & 2.308 & 0.127 & 0.458 & 3.840 & 0.065 \\
\hline without fictitious nodes & 22.286 & 6.379 & 2.863 & 10.139 & 1.619 & 0.843 \\
\hline
\end{tabular}

Table 2. The relative difference between the classical and the PD solutions for the displacement at $x=7 \mathrm{~L} / 16$ and at $t=0.19 \mathrm{~ms}$, corresponding to $\delta=L / 80$ and $m=8$.

Table 2 shows the relative difference between the PD result and the classical result for the displacement at $x=7 \mathrm{~L} / 16$ and at $t=0.19 \mathrm{~ms}$. Results are obtained with horizon size $L / 80$ and the relative grid-density factor $m=8$ used in the computations. The smallest errors are seen for the kernel generated by $n=2$ (cases C3 and L3) for both with and without fictitious nodes. We also note that using the fictitious boundaries improves the convergence rate to the classical solution. 

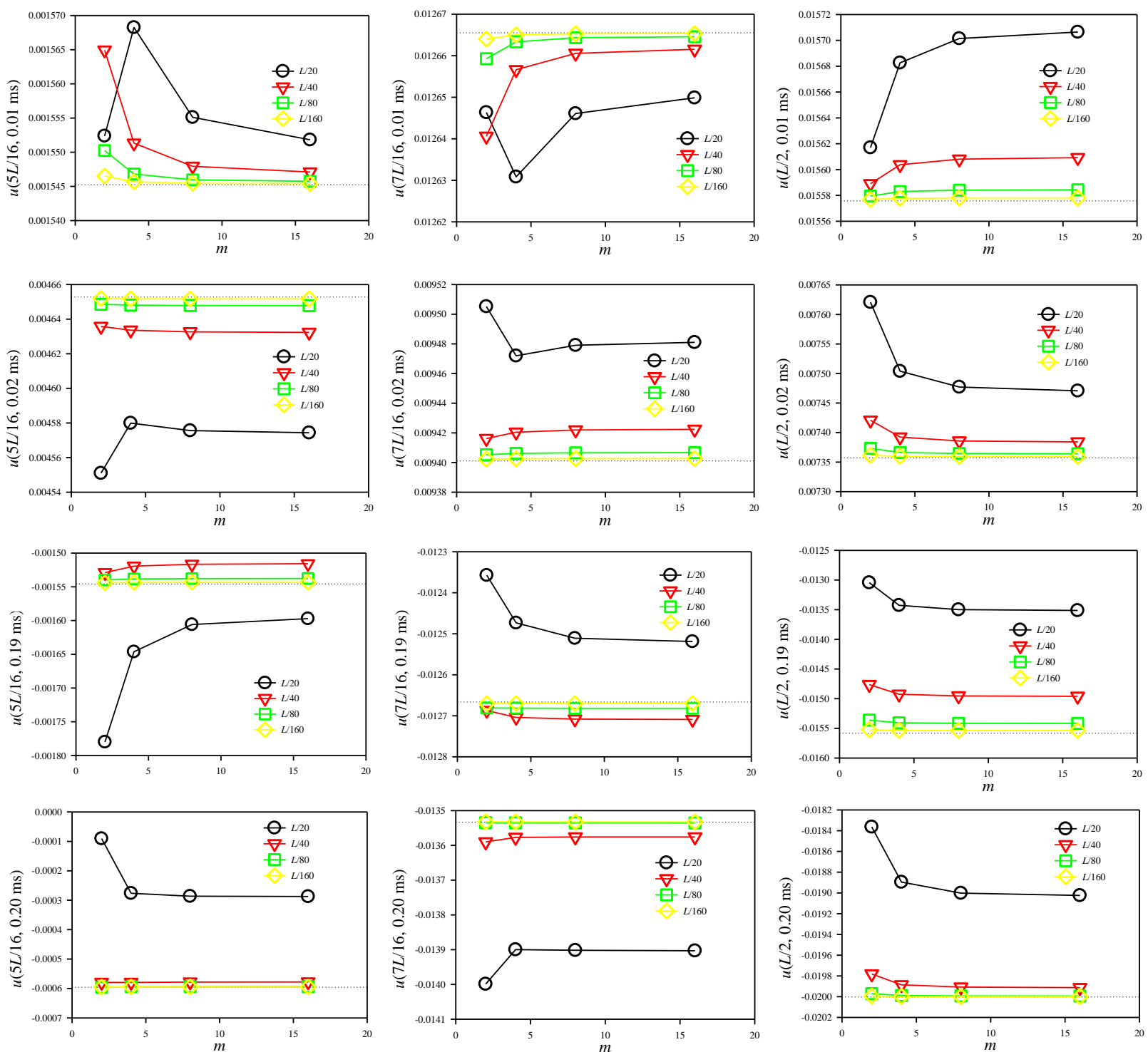

Figure 11. Solution with fictitious node method for applying the boundary condition. The $m$ convergence for kernel C3 at three different locations: $x=5 L / 16$ (left column), $x=7 L / 16$ (middle column) and $x=L / 2$ (right column), four different times: $t=0.01 \mathrm{~ms}$ (top row), $0.02 \mathrm{~ms}$ ( $2^{\text {nd }}$ row), $0.19 \mathrm{~ms}$ ( $3^{\text {rd }}$ row) and $0.20 \mathrm{~ms}$ (last row). The dotted lines represent the values of the classical solution at the corresponding locations and times.

Similar to Figure 8, Figure 11 shows the displacement $m$-convergence curves for kernel C3 at different locations and times. Before the wave bouncing off the fixed ends $(t=0.01,0.02 \mathrm{~ms})$, the boundary conditions and surface effect in the case without fictitious nodes have not affected the solution, thus, both Figures 8 and 11 show the same solution, while the solutions vary after the bouncing $(t=0.19,0.20 \mathrm{~ms})$. This behavior proves that in elasticity problems, the surface effect and the way we impose the boundary conditions is not felt uniformly or constantly at all material points. 


\section{Conclusions}

In this paper we constructed a peridynamic kernel for linear dynamic elasticity. We analyzed the behavior of the peridynamic solutions for an elastic wave in a bar with respect to the form of the kernel used, the relative grid density used, and the horizon size. One-point Gaussian quadrature was examined because of its advantages for problems in which Peridynamics is mostly used for (damage and fracture problems). We compared the behavior obtained using the constructed kernel with that from kernels postulated and used in the literature before. We found that the kernel obtained by construction (with $n=2$ in the $\frac{1}{|\hat{x}-x|^{n}}$ factor) converges (under $\delta$-convergence) to the classical solution. While $\delta$-convergence also happens for $n=0$ and 1, the limit is not the classical solution. We noted, however, that all kernels produce solutions that converge to the classical solution under $(\delta m)$-convergence. In practice, $\delta m$ convergence is (almost) never used because of its high computational cost, and $\delta$-convergence is used instead. The case for preferring a kernel with $n=2$ can be made based on the much reduced computational cost of $\delta$-convergence compared with $(\delta m)$-convergence.

Different from the diffusion case, in the wave propagation problem the boundaries are not felt immediately and their influence on the solution is felt differently at different times. We analyzed converge to the classical solution with and without surface correction (via fictitious nodes method). For the constructive kernel, the relative difference between the PD results and the classical ones is reduced by a factor larger than ten after using surface corrections. The remaining difference from the classical solution is caused by the nonlocal dispersion effect.

The convergence results of the peridynamic solutions emphasized that caution must be taken when using nonlocal models. We also warn that, occasionally, the PD solution for certain, even coarse, discretization (small $m=\frac{\delta}{\Delta x}$ ) may very closely match the exact classical solutions. However, when the discretization grid is refined (while using the same horizon size), the PD solution may start to depart from the classical one, converging to something other than the classical solution. Some results in the literature, that did not use kernels with $n=2$, given for fixed values of $\delta$ and $m$ and shown to match very well the classical results, have to be understood in this new light. Therefore, agreement of a PD numerical result with the local model does not necessarily imply that the PD solution shown is a converged one, in the $m$-convergence sense (of increasing grid density for a fixed value of the horizon). Additionally, we have shown that for the wave propagation problem, at different points in time the convergence behavior of the peridynamic model changes because of nonlocal dispersion effects, as well as due to boundary effects in cases when the surface effect is not entirely eliminated. Consequently, special care must be taken when claiming validation of PD results by specific comparisons with local model solutions. In summary, the results presented here for elasticity and those in reference [11] for diffusion, suggest that the constructive kernels be employed in peridynamic modeling.

Acknowledgements. This work has been supported in part by grants from the AFOSR MURI Center for Material Failure Prediction Through Peridynamics (program managers Drs. James 
Fillerup, Ali Sayir, David Stargel, and Fariba Fahroo), from the ARO/ARL Grant Number W911NF1010431, (program manager Dr. Larry Russell, and Dr. Asher Rubinstein), and from the ONR Award \#N00014-16-1-2173 (program manager William Nickerson).

\section{Reference}

[1] T.L. Anderson, Fracture mechanics: fundamentals and applications, CRC press, 2005.

[2] S.A. Silling, Reformulation of elasticity theory for discontinuities and long-range forces, J. Mech. Phys. Solids, 48 (2000) 175-209.

[3] S.A. Silling, M. Epton, O. Weckner, J. Xu, E. Askari, Peridynamic states and constitutive modeling, J. Elasticity, 88 (2007) 151-184.

[4] S.A. Silling, R.B. Lehoucq, Peridynamic Theory of Solid Mechanics, Advances in Applied Mechanics, Vol 44, 44 (2010) 73-168.

[5] Y.D. Ha, F. Bobaru, Characteristics of dynamic brittle fracture captured with peridynamics, Eng. Fract. Mech., 78 (2011) 1156-1168.

[6] W.K. Hu, Y.D. Ha, F. Bobaru, Peridynamic model for dynamic fracture in unidirectional fiber-reinforced composites, Comput. Methods Appl. Mech. Engin., 217 (2012) 247-261.

[7] Z. Cheng, G. Zhang, Y. Wang, F. Bobaru, A peridynamic model for dynamic fracture in functionally graded materials, Composite Structures, 133 (2015) 529-546.

[8] F. Bobaru, M. Duangpanya, A peridynamic formulation for transient heat conduction in bodies with evolving discontinuities, J. Comput. Phys., 231 (2012) 2764-2785.

[9] Z.G. Chen, F. Bobaru, Peridynamic modeling of pitting corrosion damage, J. Mech. Phys. Solids, 78 (2015) 352-381.

[10] Z.G. Chen, G.F. Zhang, B. Florin, The Influence of Passive Film Damage on Pitting Corrosion, J. Electrochem. Soc., 163 (2016) C19-C24.

[11] Z. Chen, F. Bobaru, Selecting the kernel in a peridynamic formulation: a study for transient heat diffusion, Comput. Phys. Commun., 197 (2015) 51-60.

[12] F. Bobaru, M.J. Yang, L.F. Alves, S.A. Silling, E. Askari, J.F. Xu, Convergence, adaptive refinement, and scaling in 1D peridynamics, Int. J. Numer. Meth. Eng., 77 (2009) 852-877.

[13] S. Oterkus, E. Madenci, A. Agwai, Peridynamic thermal diffusion, J. Comput. Phys., 265 (2014) 71-96.

[14] F. Bobaru, M. Duangpanya, The peridynamic formulation for transient heat conduction, Int. J. Heat Mass Transfer, 53 (2010) 4047-4059.

[15] F. Bobaru, Y.D. Ha, Adaptive Refinement and Multiscale Modeling in 2d Peridynamics, Int. J. Multiscale Com., 9 (2011) 635-659.

[16] A. Katiyar, J.T. Foster, H. Ouchi, M.M. Sharma, A peridynamic formulation of pressure driven convective fluid transport in porous media, J. Comput. Phys., 261 (2014) 209-229.

[17] P. Seleson, M.L. Parks, On the Role of the Influence Function in the Peridynamic Theory, Int. J. Multiscale Com., 9 (2011) 689-706.

[18] S.A. Silling, F. Bobaru, Peridynamic modeling of membranes and fibers, Int. J. Nonlinear Mech., 40 (2005) 395-409.

[19] S. Oterkus, E. Madenci, A. Agwai, Fully coupled peridynamic thermomechanics, J. Mech. Phys. Solids, 64 (2014) 1-23.

[20] B. Kilic, A. Agwai, E. Madenci, Peridynamic theory for progressive damage prediction in center-cracked composite laminates, Composite Structures, 90 (2009) 141-151. 
[21] W. Gerstle, S. Silling, D. Read, V. Tewary, R. Lehoucq, Peridynamic Simulation of Electromigration, Comput. Mater. Continua, 8 (2008) 75-92.

[22] W.K. Hu, Y.N. Wang, J. Yu, C.F. Yen, F. Bobaru, Impact damage on a thin glass plate with a thin polycarbonate backing, Int. J. Impact Eng., 62 (2013) 152-165.

[23] B. Kilic, E. Madenci, Prediction of crack paths in a quenched glass plate by using peridynamic theory, International Journal of Fracture, 156 (2009) 165-177.

[24] L. Malvern, Introduction to the Mechanics of a Continuous Medium, Prentice-Hall, Inc., New Jersey, 1969.

[25] S.A. Silling, M. Zimmermann, R. Abeyaratne, Deformation of a peridynamic bar, J. Elasticity, 73 (2003) 173-190.

[26] R.B. Lehoucq, S.A. Silling, Force flux and the peridynamic stress tensor, J. Mech. Phys. Solids, 56 (2008) 1566-1577.

[27] S.F. Henke, S. Shanbhag, Mesh sensitivity in peridynamic simulations, Comput. Phys. Commun., 185 (2014) 181-193.

[28] D. Dipasquale, M. Zaccariotto, U. Galvanetto, Crack propagation with adaptive grid refinement in 2D peridynamics, International Journal of Fracture, 190 (2014) 1-22.

[29] X.C. Tian, Q. Du, Analysis and Comparison of Different Approximations to Nonlocal Diffusion and Linear Peridynamic Equations, SIAM J. Numer. Anal., 51 (2013) 3458-3482.

[30] X.C. Tian, Q. Du, Asymptotically compatible schemes and applications to robust discretization of nonlocal models, SIAM J. Numer. Anal., 52 (2014) 1641-1665.

[31] M. Duangpanya, A Peridynamic Formulation for Transient Heat Conduction in Bodies with Evolving Discontinuities, Ph.D. thesis, University of Nebraska - Lincoln, 2011.

[32] Q. Le, F. Bobaru, Surface corrections in peridynamic models in elasticity and around fracture, In review, (2016). 